\title{
Electron and hole wave functions in self-assembled quantum rings
}

\author{
J. A. Barker \\ Optoelectronics Group, Cavendish Laboratory, University of Cambridge, Cambridge CB3 OHE, United Kingdom \\ R. J. Warburton \\ Department of Physics, School of Engineering and Physical Sciences, Heriot-Watt University, Edinburgh EH14 4AS, United Kingdom \\ E. P. O’Reilly \\ NMRC, University College, Lee Maltings, Prospect Row, Cork, Ireland
}

(Received 19 December 2002; revised manuscript received 1 August 2003; published 28 January 2004)

\begin{abstract}
We use a plane-wave technique to study the electron and hole wave functions in self-assembled InGaAs quantum rings, investigating the influence on the electron-hole overlap of variations in the ring shape and composition profiles, and including the effects of built-in strain and the piezoelectric potential. Lateral variation of the ground-state electron and hole wave functions in a perfect ringlike structure is observed as a consequence of the piezoelectric potential, which has minima and maxima on the (110) and (1 $\overline{1} 0)$ planes passing through the center of the ring. We find for rings with reflection symmetry in the vertical direction that although the average electron and hole positions are equal, there is a significant vertical separation of the electron and hole peak probability densities in the (110) and (1 $\overline{1} 0)$ planes. A net vertical separation of the electron and hole may occur either through the piezoelectric potential when the (110) and (1 $\overline{1} 0)$ planes are no longer equivalent, or through strain effects when there is a shape or composition asymmetry in the [001] direction. Comparison of the theoretical predictions with experimental data confirm the presence of a large asymmetry in the ring profile both in the vertical direction, where the ring is deduced to be volcanolike, and also in the growth plane, where the rings are elongated along the [1 $\overline{1} 0]$ direction.
\end{abstract}

DOI: 10.1103/PhysRevB.69.035327 PACS number(s): 78.67.Bf, 73.21.-b, 73.40.Rw, 78.66.Fd

\section{INTRODUCTION}

Three-dimensional confinement of charge carriers in quantum dots makes these nanostructures extremely attractive for use in photonic devices and for quantum computing applications. ${ }^{1}$ Of particular interest are self-assembled quantum dots which are free of defects and are therefore efficient optical emitters. $^{2,3}$ It is now clear that a wide variety of different dot structures can be self-assembled by subtle changes in the growth conditions. For instance, InAs quantum dots on GaAs grown at low temperatures are small and have a pyramidal character ${ }^{4}$ whereas InAs quantum dots grown at higher temperatures are larger with a lenslike shape. ${ }^{5}$ The detailed shape and composition profile of the dots has important consequences for the optical properties. This has been demonstrated recently for InGaAs dots where an indium concentration profile, from indium poor at the base to indium rich at the apex, ${ }^{6}$ causes the center of gravity of the hole wave function to lie above that of the electron, giving a net negative permanent dipole moment along the growth direction. ${ }^{7}$

A notable development in the self-assembly of semiconductor nanostructures has been the growth of quantum rings. ${ }^{8}$ The rings are formed from large InAs quantum dots by partially covering the quantum dots with GaAs to destabilize them. During an annealing step at the growth temperature, a remarkable change in topology occurs, such that the original dots acquire a hole in their center and take on a ringlike shape. The ring formation is presently not understood in detail, but it has been suggested that it arises through a dewetting process. ${ }^{9,10}$ The rings have quite different electronic properties compared to the original dots, ${ }^{11-16}$ including in particular a large and positive permanent dipole moment, with the electron center of gravity about $1 \mathrm{~nm}$ above the hole center of gravity. ${ }^{15}$

The discovery of the self-assembly of quantum rings has initiated a number of theoretical studies into their properties. The significant differences from lithographically defined rings are that the ring confines both electron and hole, ${ }^{14}$ not just the electron, and that it is possible to load a selfassembled ring with a small number of electrons starting from zero occupancy. ${ }^{11}$ A perfect ring exhibits quantum interference effects in a magnetic field, resulting in AharanovBohm-like oscillations in the magnetization as a function of applied magnetic field. ${ }^{17,18}$ There is evidence in selfassembled rings for the first Aharanov-Bohm oscillation from vertical tunneling experiments. ${ }^{11}$ The possibility of an Aharanov-Bohm effect for an exciton has been considered. An exciton is a neutral particle and so at first sight no phaserelated effects are expected. However, the exciton is a composite particle, and this allows for the possibility of weak phase-related effects. ${ }^{19,20}$ For a one-dimensional ring, both the exciton energy and oscillator strength oscillate with magnetic field, although the effect is exponentially small. ${ }^{19,20}$ The width of self-assembled rings is a significant fraction of their radius and so this needs to be taken into account. For finite width rings, the general consensus from theoretical investigations is that the Aharanov-Bohm effects in the ground-state exciton disappear, ${ }^{21-23}$ but there are flux-related oscillations in the energies of the excited states. ${ }^{22,23}$ The result on the ground state has been recently confirmed by photoluminescence experiments on rings defined by etching a ring shape in a semiconductor quantum well. ${ }^{24}$ In these experiments, 
there is no evidence for an Aharanov-Bohm effect in the neutral exciton, but the charged exciton displays a fluxrelated effect largely through the pronounced AharanovBohm effect of the electron left behind after recombination. ${ }^{24}$ Models have been developed to account for various factors in semiconductor quantum rings, for instance the eccentricity, ${ }^{25}$ the effective mass discontinuity in the conduction band, ${ }^{26}$ and the valence band structure, ${ }^{27}$ and also the behavior in a lateral electric field which can enhance the Aharanov-Bohm effect by delocalizing the electron and hole. ${ }^{28}$ However, all these models, and also those developed for mesoscopic systems, ${ }^{18}$ assume that the lateral potential depends only on the radial coordinate and not on the azimuth angle. We point out here that for self-assembled quantum rings, this assumption is no longer valid. The piezoelectric field reduces the symmetry such that the potential depends on the azimuth angle, and for realistic parameters we find that this can lead to significant localization of the wave functions, particularly for the hole.

In the present paper, we present calculations of the electron and hole wave functions in self-assembled semiconductor quantum rings, considering a variety of different ring shapes and composition profiles. We use a carefully chosen one-band effective mass Hamiltonian to investigate the electron and hole ground-state wave functions, demonstrating how the symmetry of the wave functions follows the piezoelectric potential distribution and strain fields of the inclusion. To elucidate some of the key points and to allow a comparison with facetted quantum dots, we consider initially both circular and square lateral profiles, both with a rectangular, reflection-symmetric vertical profile. We consider how the wave functions are modified if we break the reflection symmetry of the ring in the vertical direction by introducing an indium concentration gradient, and for a circular lateral profile, we investigate the effect of breaking the cylindrical symmetry by elongating the ring along the $[1 \overline{1} 0]$ direction. These results show that the piezoelectric field and the biaxial strain distribution each play a crucial role in determining the final form of the electron and hole ground-state wave functions. We then go on from these model calculations to consider more realistic ring shapes. General trends for the ground-state wave functions can be deduced, and these allow us to conclude that the spectroscopy results on selfassembled quantum rings are consistent with the directly measured shape and symmetry of uncapped rings.

\section{METHOD}

Following Ref. 29, we solve Schrödinger's equation for a system of semiconductor nanostructures using a plane-wave envelope function Hamiltonian. Assuming a threedimensional superlattice of rings with a unit cell size of $2 L_{x} \times 2 L_{y} \times 2 L_{z}$, the energy eigenvalues $E_{n}$ for the system are given by

$$
\sum_{\mathbf{k}} \bar{H}_{\mathbf{k}, \mathbf{k}^{\prime}} A_{\mathbf{k}}^{n}=E_{n} \sum_{\mathbf{k}} A_{\mathbf{k}}^{n}
$$

where $A_{\mathbf{k}}^{n}$ is the coefficient for the plane wave of the $n$th eigenvalue whose wave vector $\mathbf{k}=\left(k_{x}, k_{y}, k_{z}\right)$ $=\pi\left(a / L_{x}, b / L_{y}, c / L_{z}\right) . a, b$, and $c$ take integer values. The matrix $\bar{H}_{\mathbf{k}, \mathbf{k}^{\prime}}$ is the Fourier transform of the Hamiltonian $\hat{H}(\mathbf{r})$, given by

$$
\bar{H}_{\mathbf{k}, \mathbf{k}^{\prime}}=\int \frac{d^{3} \mathbf{r}}{8 L_{x} L_{y} L_{z}} \exp \left(-i \mathbf{k}^{\prime} \cdot \mathbf{r}\right) \hat{H}(\mathbf{r}) \exp (i \mathbf{k} \cdot \mathbf{r}),
$$

with $\mathbf{r}=(x, y, z)$. Use of a plane-wave basis set allows us to find analytic expressions for complex potential distributions such as the strain and piezoelectric distributions for the nanostructure shapes of interest. ${ }^{30}$ For example, the Fourier transform $\tilde{\varepsilon}_{i j}$ of the strain tensor components $\varepsilon_{i j}$ is given by $\left(\mathbf{k}^{\prime \prime} \neq 0\right)$

$$
\tilde{\varepsilon}_{i j}=\tilde{\chi}\left(\mathbf{k}^{\prime \prime}\right) \varepsilon_{0}\left[\delta_{i j}-\left(\frac{C_{11}+2 C_{12}}{C_{11}}\right) \frac{k_{i}^{\prime \prime} k_{j}^{\prime \prime}}{\left|\mathbf{k}^{\prime \prime}\right|^{2}}\right],
$$

where $\mathbf{k}^{\prime \prime}=\mathbf{k}-\mathbf{k}^{\prime}, \tilde{\chi}\left(\mathbf{k}^{\prime \prime}\right)$ is the Fourier transform of the ring characteristic function, $C_{11}$ and $C_{12}$ are the elastic constants for the material, and $\varepsilon_{0}$ is the lattice mismatch of the ring and barrier materials (6.7\% for InAs in GaAs). An extension of the analysis of Ref. 30 to the piezoelectric potential $d_{p z}$ gives the Fourier transform $\widetilde{d}_{p z}$ in terms of the piezoelectric constant $e_{14}$. We have

$$
\widetilde{d}_{p z}=\frac{-6 i e_{14} \varepsilon_{0}}{\epsilon_{0} \epsilon_{r}} \tilde{\chi}\left(\mathbf{k}^{\prime \prime}\right)\left(\frac{C_{11}+2 C_{12}}{C_{11}}\right) \frac{k_{x}^{\prime \prime} k_{y}^{\prime \prime} k_{z}^{\prime \prime}}{|\mathbf{k}|^{4}} .
$$

We therefore treat the strain and the piezoelectric potential on an equal footing, as both are functions of $\tilde{\chi}(\mathbf{k})$. The repeat distances $2 L_{x}, 2 L_{y}$, and $2 L_{z}$ are chosen to be large enough that there is a negligible interaction between one ring and its neighbor.

We take the band gaps and effective masses for GaAs (InAs) to be 1.519 (0.42) eV and 0.067 (0.023), respectively. The conduction-(valence-) band edges between unstrained InAs and unstrained GaAs are offset by $0.834 \mathrm{eV}(0.265 \mathrm{eV})$. For the $\operatorname{In}_{x} \mathrm{Ga}_{1-x}$ As alloy, we assume that the energy gap, the band offsets and the inverse effective masses vary linearly with $x$. All other parameters are listed in Ref. 29. We have shown previously, using the example of a pyramidal dot shape, that this method gives good agreement with the electron and heavy hole ground-state energies calculated with a more accurate eight-band $\mathbf{k} \cdot \mathbf{P}$ model ${ }^{29}$ This result is expected for the electron; for the heavy hole, it arises because the heavy hole is largely decoupled from the other valence states because of quantum confinement and the large biaxial strain. We can therefore use the method with confidence to calculate the electron and heavy-hole ground states in quantum rings. We concentrate here on the form of the wave functions for various ring shapes and composition profiles and for this our method is perfectly adequate. As we show, the uncertainties in the exact shape and composition profiles of the rings introduce far more variations in the results than would arise from different levels of sophistication in the theory. 

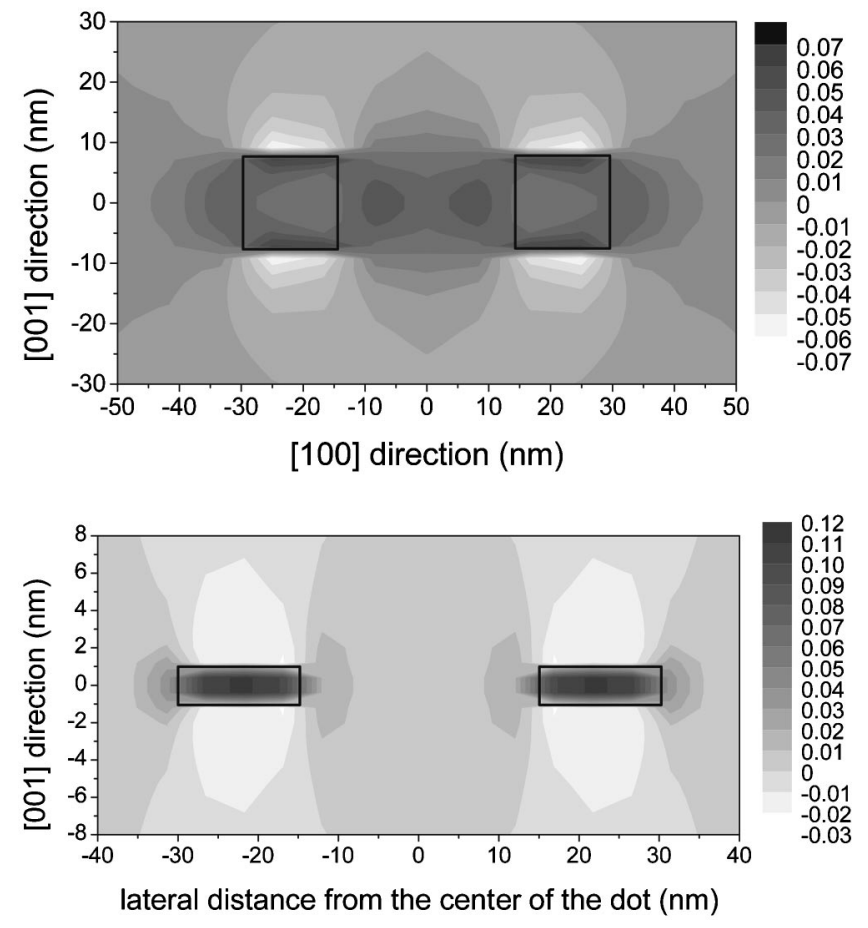

FIG. 1. Biaxial strain distribution as a function of position on the (010) plane through the ring center for a disclike InAs/GaAs quantum ring of outer diameter $60 \mathrm{~nm}$, inner diameter $30 \mathrm{~nm}$, and height $15 \mathrm{~nm}$ (top) and for a similar ring of height $2 \mathrm{~nm}$ (bottom). The gray scales indicate the strain value (in dimensionless units).

\section{GENERAL RESULTS FOR ELECTRON AND HOLE WAVE FUNCTIONS IN QUANTUM RINGS}

We consider initially a ring with a circular lateral profile and cylindrical symmetry. The ring is assumed to be pure InAs, embedded in GaAs. Because of the large lattice mismatch between InAs and GaAs, the ring is highly strained. Figure 1 plots the biaxial strain $\left[\varepsilon_{a x}=\varepsilon_{z z}-\left(\varepsilon_{x x}+\varepsilon_{y y}\right) / 2\right]$ distribution for two cylindrical rings, both with outer diameter $60 \mathrm{~nm}$ and inner diameter $30 \mathrm{~nm}$. One ring has vertical thickness $15 \mathrm{~nm}$ (top), the other $2 \mathrm{~nm}$ (bottom). It can be seen that for the $15 \mathrm{~nm}$ thick ring, the biaxial strain is negative (as defined here) in the regions immediately above and below the ring, taking large positive values in the GaAs barrier material at the ring center. The biaxial strain is more concentrated inside the ring for the $2 \mathrm{~nm}$ thick ring, but also here there is some biaxial strain in the core region.

The large strain in the quantum rings generates a piezoelectric field. Figure 2 contains contour plots of the piezoelectric potential on the (110) plane for two rings with square lateral profiles. We take a square lateral profile in order to facilitate an immediate comparison with the results for a cubic dot for which there are eight piezoelectric extrema, one close to each corner [see Fig. 1(b) of Ref. 31]. The most striking feature of Fig. 2(a) is that, in addition to the set of eight piezoelectric extrema near to the eight outer corners of the ring, there is a second set of potential minima and maxima at the inner edges. These additional piezoelectric extrema arise from the presence of the GaAs core. We note that a cylindrical ring, as in Fig. 1, has qualitatively the same
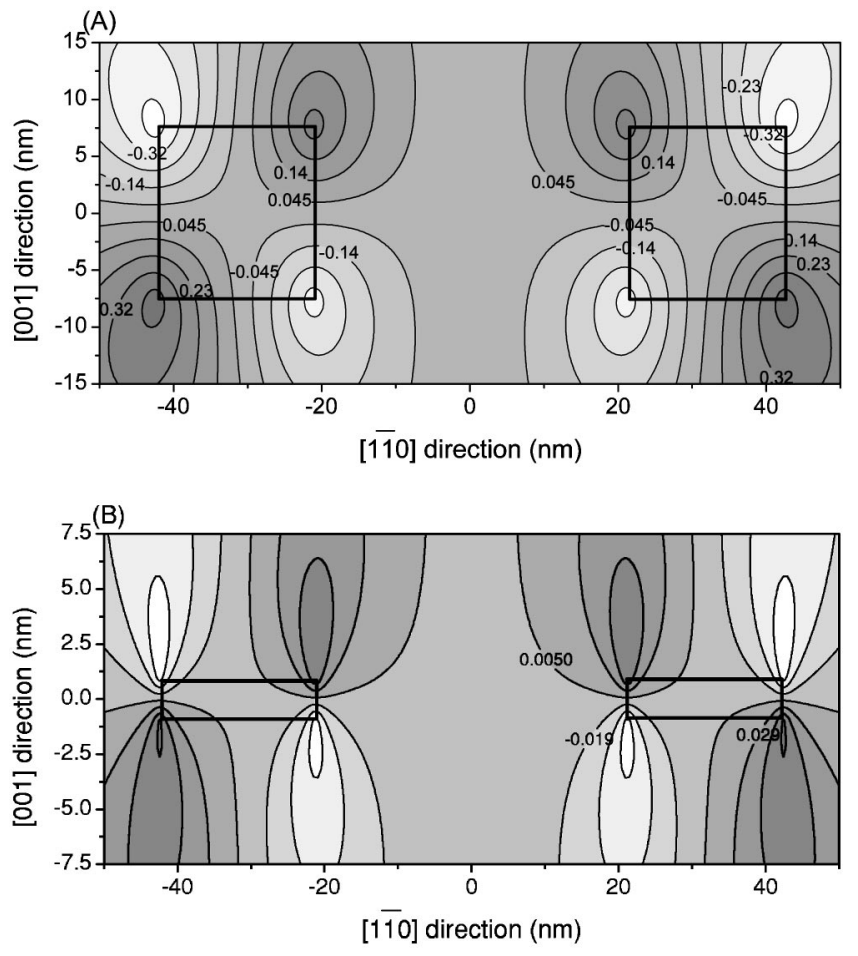

FIG. 2. Piezoelectric potential distribution, as a function of position on the (110) plane through the ring center, for InAs/GaAs quantum rings with a square profile. (a) Ring thickness $15 \mathrm{~nm}$ with lateral dimensions $60 \mathrm{~nm}$ for the outer square length, $30 \mathrm{~nm}$ for the inner square length. (b) Ring thickness $2 \mathrm{~nm}$ with lateral dimensions also $60 \mathrm{~nm}$ for the outer square length, $30 \mathrm{~nm}$ for the inner square length. The thick solid lines show the interface between InAs and GaAs and the labels of the contours are in volts. The potential energy in electron volt experienced by an electron (hole) is found by multiplying the piezoelectric potential by $-1(+1)$.

distribution of piezoelectric potential as the rings in Fig. 2. The reason for this is that the piezoelectric potential depends on the lateral crystal direction such that, even for a ring with a shape invariant to an arbitrary rotation, the piezoelectric field introduces a two-fold symmetry to the potential.

For dots, the piezoelectric potential extrema lie well outside the dots, whereas the electron and hole wave functions are quite strongly localized inside the dots, such that the piezoelectric potential has a negligible effect on the electron and hole ground-state wave functions. ${ }^{29,31,32}$ Conversely, for the quantum ring of Fig. 2(a), the overlap of the electron and hole wave functions with the central piezoelectric potential is significantly larger than the overlap with the outer piezoelectric potential, so that we can expect a more pronounced piezoelectric deformation of the wave functions for a ring than for a dot of similar dimensions. In fact, since the piezoelectric potentials are large for a ring with these dimensions, with peak values of $\pm 0.4 \mathrm{~V}$, the position and shape of the ground-state electron and hole wave functions are heavily influenced by the piezoelectric potential for this structure. Figure 2(b) plots the piezoelectric potential for a ring with the same lateral dimensions as the ring in Fig. 2(a), but with a height reduced to $2 \mathrm{~nm}$. The smaller volume of this ring reduces the magnitude of the piezoelectric extrema to less 
than $0.1 \mathrm{~V}$ in this case. We also note that the high aspect ratio of this ring deforms the piezoelectric potential, pushing the extrema out from the corners of the ring, which reduces the overlap between the piezoelectric potential and the groundstate electron and hole wave functions. The piezoelectricinduced deformations of the ground-state wave functions are considerably less for the ring in Fig. 2(b) than for the ring in Fig. 2(a), but nevertheless the piezoelectric potential still has a significant influence.

In addition to the piezoelectric term in the Hamiltonian, the heavy-hole wave functions are influenced by the biaxial strain distribution. In particular, the negative biaxial strain in the ring core lowers the potential barrier for the heavy holes allowing the heavy-hole wave function to spread inwards. This increases the effective volume seen by the holes and shifts the ground-state heavy-hole energy level away from the GaAs band edge. The mean lateral radius $\sqrt{\left|\left\langle\psi_{h}\left|\hat{x}^{2}+\hat{y}^{2}\right| \psi_{h}\right\rangle\right|}$ of the ground-state hole wave function $\psi_{h}$ is therefore decreased. The electron wave function, $\psi_{e}$, is influenced only by the hydrostatic strain and not by the biaxial strain, so that this attraction into the central core is absent for the electrons. Consequently, the electron radius $\sqrt{\left|\left\langle\psi_{e}\left|\hat{x}^{2}+\hat{y}^{2}\right| \psi_{e}\right\rangle\right|}$ is larger than the hole radius for this particular form of quantum ring. However, for the $2 \mathrm{~nm}$ thick ring, the biaxial strain fields do not extend particularly far into the central core of the ring for this shape. We can therefore anticipate that for the real self-assembled quantum rings, which have a small vertical extent and a high aspect ratio, the biaxial strain is a relatively unimportant mechanism for introducing a lateral separation of the electron and hole wave functions. Nevertheless, as we show by considering a variety of ring shapes in the following section, the biaxial strain can play a significant role in introducing a vertical separation of the electron and hole wave functions in rings which have a shape or composition asymmetry in the vertical (growth) direction.

We now turn to the electron and hole wave functions in quantum rings, considering first the role of the piezoelectric potential in rings which are symmetric in the vertical direction. Figures 3(a) and 3(b) are birds-eye views of the groundstate electron and hole probability densities $\left|\psi_{e(h)}\right|^{2}$ for an InAs/GaAs quantum ring with cylindrical symmetry. The outer diameter is $60 \mathrm{~nm}$, the inner diameter $30 \mathrm{~nm}$, and the height $2 \mathrm{~nm}$. Both the ground-state electron wave function $\psi_{e}$ and hole wave function $\psi_{h}$ are continuous around the ring. Although the cylindrical symmetry of the inclusion results in an electron-hole dipole $d=\left|\left\langle\psi_{h}|\hat{z}| \psi_{h}\right\rangle\right|$ $-\left|\left\langle\psi_{e}|\hat{z}| \psi_{e}\right\rangle\right|$ equal to zero, the peak probability densities of the ground state carriers in this ring are vertically separated by the piezoelectric potential on the (110) and (1 $\overline{1} 0)$ planes through the ring center, decreasing the wave function overlap $\left|\left\langle\psi_{e} \mid \psi_{h}\right\rangle\right|$, which takes a value of 0.854 for this ring, compared with a value much closer to 1 for a quantum dot of similar dimensions. The piezoelectric-induced deepening of the electron and hole potential energy wells also increases the ground-state confinement energies measured relative to the GaAs conduction and valence band edges, respectively, by several $\mathrm{meV}$, with the greater shift in the heavy-hole ground state.

At this point, we consider the effects of reducing the symmetry in the vertical direction. As an example, we introduce a vertical composition gradient as has been discussed for quantum dots. ${ }^{7}$ This is not meant to be a realistic representation of a real ring. Rather, we introduce the composition gradient in order to break the vertical symmetry while keeping the ring volume the same. Figures 3(c) and 3(d) are birds-eye views of the ground-state probability densities for an $\operatorname{In}_{x} \mathrm{Ga}_{1-x}$ As ring with the same geometry as for Figs. 3(a) and $3(\mathrm{~b})$, but with a composition varying linearly with $z$ from $x=0$ at the base to $x=1$ at the top of the ring. In the absence of the piezoelectric field, the ground-state electron and hole wave functions would be uniform and continuous around the ring. This is clearly not the case here, with the hole wave function localized in one particular plane [Fig. 3(d)]. This arises because the piezoelectric potential introduces a $\theta$-dependent potential [ $\theta$ is the azimuth angle in the $(x, y)$ plane with the origin at the ring center]. In the particular case of Figs. 3(c) and 3(d), the small ring volume results in a ground-state electron wave function which is spread throughout the ring, whereas the large hole effective mass causes the hole wave function to shift towards the top of the ring because of the indium concentration gradient. This not only introduces a vertical electron-hole dipole moment, but it also increases the overlap between the hole wave function and the piezoelectric potential minima on the $(1 \overline{1} 0)$ plane towards the inside of the ring, so that the hole wave function becomes localized. The important point is that the vertical asymmetry in the structure of the ring leads to a pronounced change in the lateral symmetry in the carrier wave functions, particularly for the hole.

A further source of symmetry breaking is a possible departure from a perfectly circular shape in the $(x, y)$ plane. Figures 3(e) and 3(f) show the electron and hole ground-state probability densities for an InAs quantum ring which has been stretched to an outer diameter of $72 \mathrm{~nm}$ on the (110) plane and squeezed to an outer diameter of $48 \mathrm{~nm}$ on the (1 $1 \overline{1} 0)$ plane. In this case, both electron and hole wave functions are localized by a combination of the piezoelectric potential and the ring shape itself, since there is a larger volume of ring material centered on the (110) plane than on the ( $1 \overline{1} 0)$ plane. Even in the absence of any vertical asymmetry, the lateral asymmetry introduces an electron-hole dipole moment in this case because the piezoelectric field tends to separate the electron and hole, pulling the electron to the top of the elongated ring and the hole to the bottom.

The calculations so far have shown that the piezoelectric potential is important for quantum rings because it introduces a $\theta$-dependent potential. For all but the smallest quantum rings, this is sufficient to localize the heavy holes which have a large effective mass along the [001] direction. In detail however, the structures considered so far cannot be accurate representations of real quantum rings as they do not reproduce the physical properties. We therefore extend the calculations to other shapes and composition profiles in order 

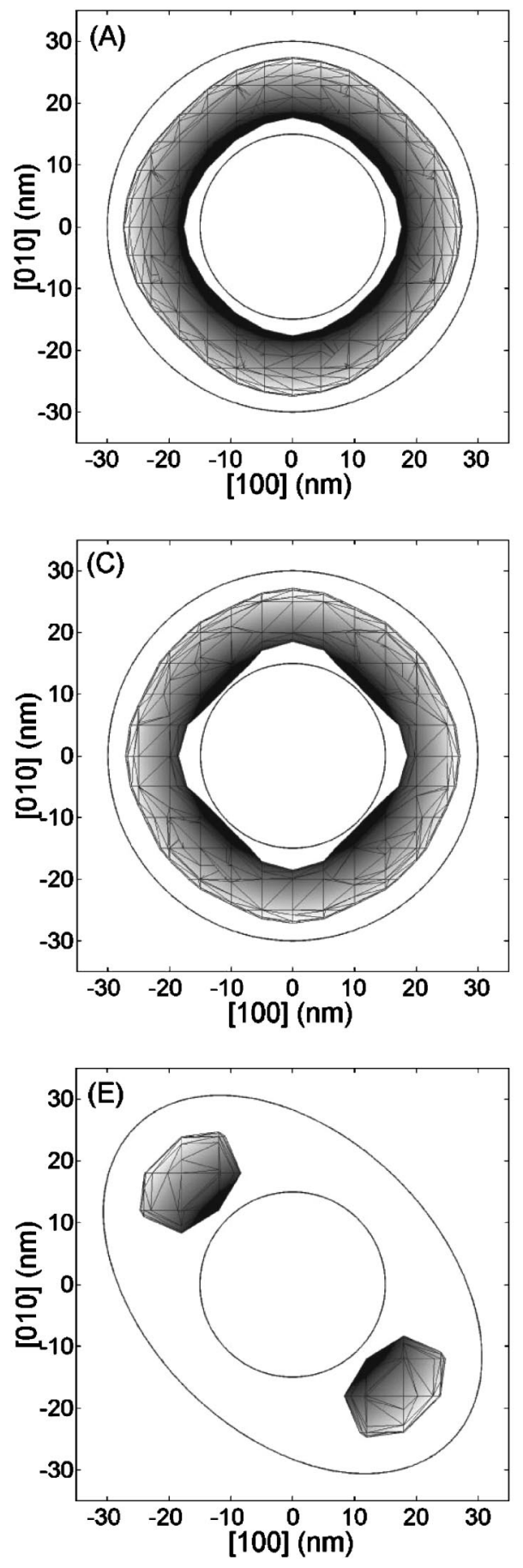
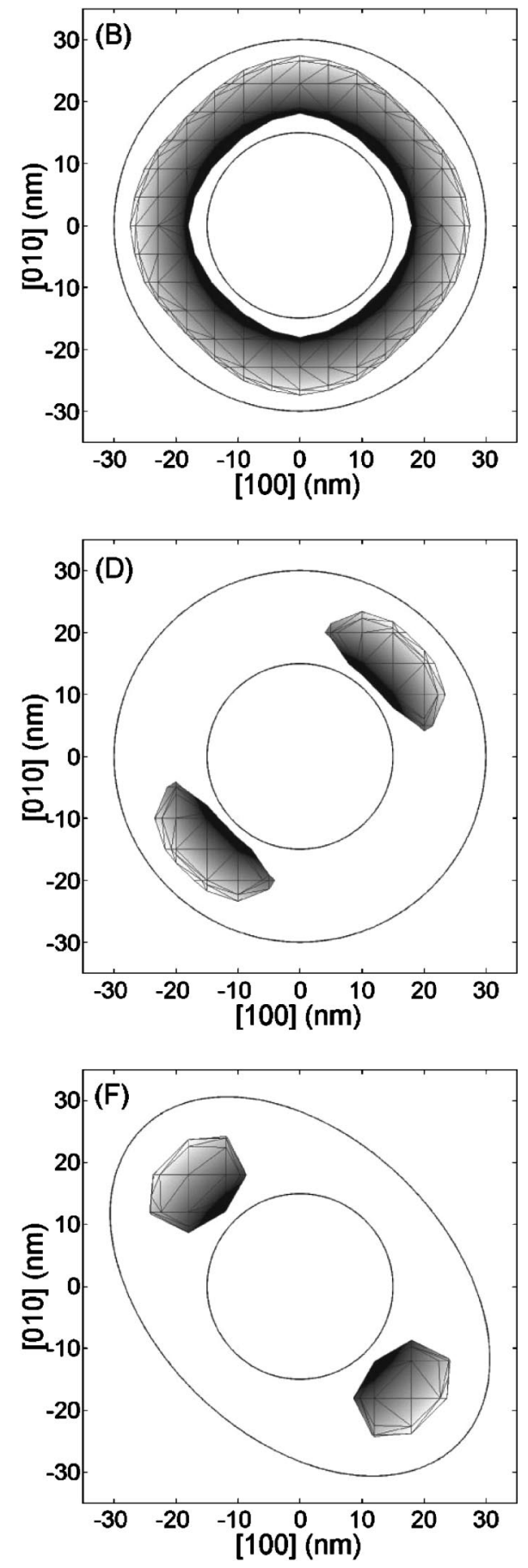

FIG. 3. Surface plots (top down) of carrier probability densities $|\psi(\mathbf{r})|^{2}$, plotting the contour at $50 \%$ of the peak probability density. (a) The ground-state electron wave function in a cylindrical InAs/GaAs quantum ring of outer diameter $60 \mathrm{~nm}$, inner diameter 30 $\mathrm{nm}$, and height $2 \mathrm{~nm}$. (b) The ground-state heavy-hole wave function for the ring considered in (a). (c) The ground-state electron wave function in a quantum ring with the same geometry as (a), but with an $\mathrm{In}_{x} \mathrm{Ga}_{1-x}$ As composition profile which varies from $x=0$ at the base of the ring to $x=1$ at the top of the ring. (d) The groundstate heavy-hole wave function for the ring considered in $(c)$. (e,f) The ground-state electron and hole wave functions in a quantum ring with a similar geometry and composition profile to (a), but with an outer diameter of $72 \mathrm{~nm}$ on the (110) plane through the ring center and an outer diameter of $48 \mathrm{~nm}$ on the $(1 \overline{1} 0)$ plane through the ring center. to explore the connection between the experimental results and the calculations.

\section{COMPARISON OF CALCULATED WAVE FUNCTIONS WITH EXPERIMENTAL RESULTS ON SELF- ASSEMBLED QUANTUM RINGS}

We consider all available experimental data on selfassembled quantum rings, namely, measurements of the topography with atomic force micrography (AFM), ${ }^{12}$ capacitance-voltage spectroscopy for information on the electron wave functions, ${ }^{11}$ and single quantum ring photolu- minescence in a vertical electric field as a measurement of the typical electron-hole dipole moment. ${ }^{15}$ Figure 1 of Ref. 9 and Fig. 1 of Ref. 12 show atomic force micrographs of self-assembled $\operatorname{In}_{x} \mathrm{Ga}_{1-x}$ As quantum rings where it is clear that each nanostructure has a pronounced hole at its center. Figure 4 shows a cross-sectional height profile through the center of a typical quantum ring. It is striking that the hole in the center extends to a deeper level than the material surrounding the quantum ring, a consequence of the expulsion of material during ring formation. ${ }^{12}$ The atomic force micrographs are recorded on a surface ring, whereas the capacitance-voltage and optical spectroscopy measurements 


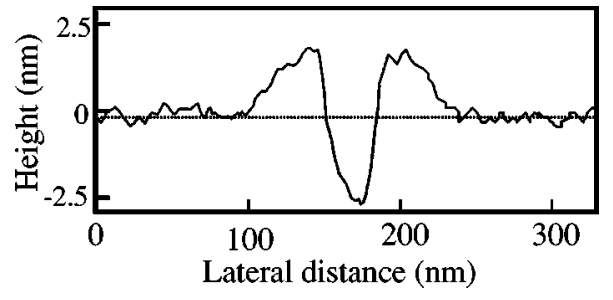

FIG. 4. The cross section through the center of an uncapped self-assembled quantum ring taken with an atomic force microscope.

are all carried out on buried rings. There is now strong evidence from cross-sectional scanning tunneling microscopy that burying the rings with GaAs after their formation does not destroy the ring shape. ${ }^{33}$ The reason for this is that the quantum rings are given sufficient time to form after which the GaAs cap layer is deposited relatively quickly, not allowing significant further changes in shape to occur. From the point of view of calculating the wave functions, it is not $a$ priori clear how the complicated shape in Fig. 4 can be best described, particularly as the composition profile is unknown. Also, it is clear in Fig. 4 that it is very misleading to describe the height as $2 \mathrm{~nm}$, the approximate height between the top of the ring and the background substrate level. The real height is better described by 4 or $5 \mathrm{~nm}$, the height between the top of the ring and the crater in the center. We suggest that the topology can be represented with the shape shown in Fig. 5, a quantum ring whose height decreases linearly from a maximum value at the inner rim of the ring to zero at the outer radius. In all cases, we assume that the material surrounding the ring, including that in the central core, is pure GaAs. As the steep interfaces between the central core and the ring itself are very unlikely in real structures, we have smeared the interfaces by including diffusion in the calculations with a diffusion length of $5 \mathrm{~nm}$. We find that the general form of the wave functions does not change on smearing the interfaces, and the quantitative changes are insignificant relative to the large changes we find in exploring the parameter space. For simplicity therefore, we present the results with the hard boundaries.

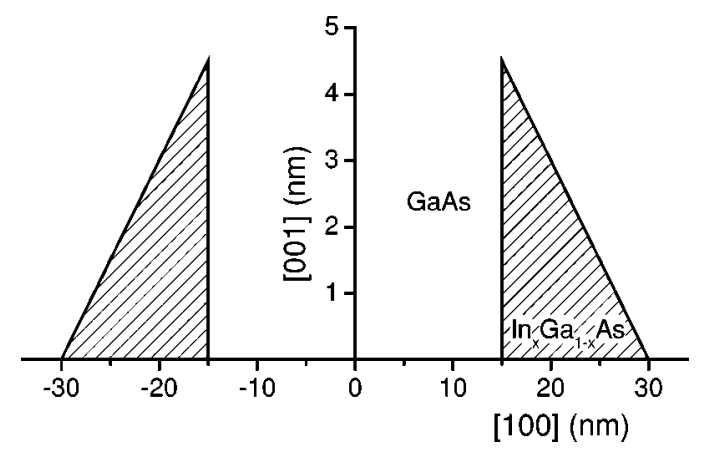

FIG. 5. Quantum ring profile assumed in the calculations. The ring has a height which decreases linearly from the inner rim to the outer edge. The ring is either pure InAs or a composition profile is introduced with the indium concentration $x$ decreasing linearly from $x=1$ at the inner rim to $x=0$ at the outer edge. The inner radius is $15 \mathrm{~nm}$, the height $4.5 \mathrm{~nm}$, and the outer radius either 30 or $22.5 \mathrm{~nm}$.
For self-assembled quantum rings, capacitance spectroscopy has been used to monitor changes in the ground-state energy as a function of magnetic field, and a kink in the plot of charging voltage against magnetic field was observed around $8 \mathrm{~T}^{11,12}$ These experiments probe what is expected to be the most notable difference between the electronic structure of a quantum dot and a quantum ring. For an electron in a quantum dot, the ground state maintains an $s$-like symmetry at all magnetic fields. For a perfect quantum ring, this is no longer the case. When one flux quantum threads the central hole, the ground state becomes $p$-like, and further changes to the ground-state angular momentum occur as additional flux quanta penetrate the ring. ${ }^{17}$ The kink in the charging voltage has been interpreted as the transition from an $s$-like to a $p$-like ground state because the magnetic field for the transition is consistent with the size of the central hole. This experiment is therefore a strong indication that the electron wave function in a typical self-assembled quantum ring propagates around the ring, rather than being localized in just one part of the structure. While the hole may be localized in one part of the ring, we look therefore in our simulations for electron wave functions that are delocalized.

In order to interpret cross-gap optical properties it is strictly necessary to include in addition to the strain and piezoelectric field also the Coulomb interaction between the electron and hole. However, in this work we are interested in the general features such that we neglect the Coulomb interaction. The justification for this is that the hole localization we find in the theory is so strong that also the first excited hole state is localized in the same plane as the hole ground state. The self-assembled quantum rings exhibit strong photoluminescence with a typical recombination lifetime of just less than a nanosecond. ${ }^{34}$ We interpret this to mean that there is a strong overlap between the electron and hole wave functions and this is used to guide the simulations. Through single quantum ring experiments in a vertical electric field, it has been found that excitons in self-assembled quantum rings possess permanent dipole moments, i.e., even with no applied bias, the average position of the electron and average position of the hole are different. ${ }^{15}$ The dipole moments are both opposite in sign and larger in magnitude than those of InAs quantum dots. For the rings, the mean position of the hole is experimentally determined to lie below the mean position of the electron by typically $1 \mathrm{~nm}$.

The calculated electron and hole wave functions presented so far cannot be accurate representations of real quantum rings because the electron-hole dipole moments are either zero [Figs. 3(a) and 3(b)], or of the wrong sign [Figs. 3(c) and 3(d)], or far too small [Figs. 3(e) and 3(f)]. In fact, for a variety of ring shapes and composition profiles, we find that it is not possible to calculate a dipole moment as large as $1 \mathrm{~nm}$ with a vertical ring height of just $2 \mathrm{~nm}$, simply because the boundaries of the quantum ring do not allow for large electron and hole separations. This is a strong argument in favor of a much larger ring height.

The first structure we present is a pure InAs quantum ring with a circular lateral profile with outer diameter $60 \mathrm{~nm}$, inner diameter $30 \mathrm{~nm}$, and maximum height $4.5 \mathrm{~nm}$, whose height decreases linearly from a maximum value at the inner 

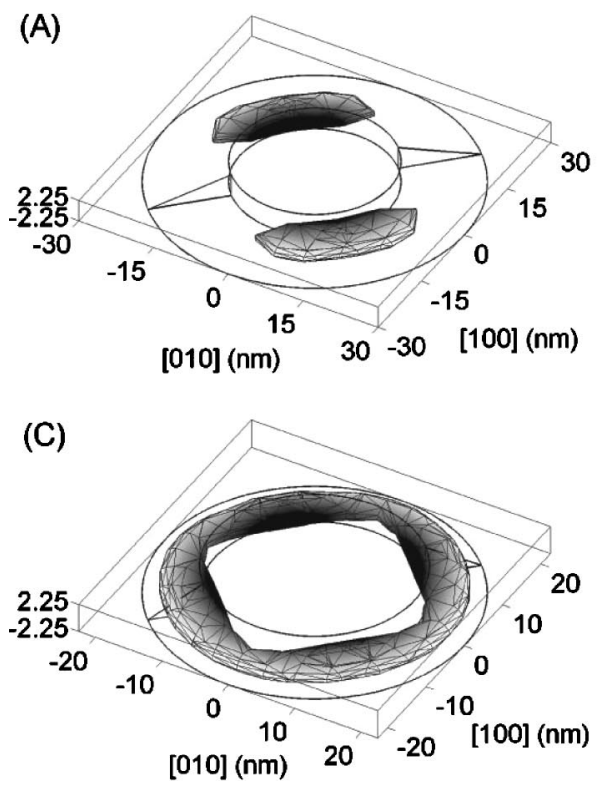
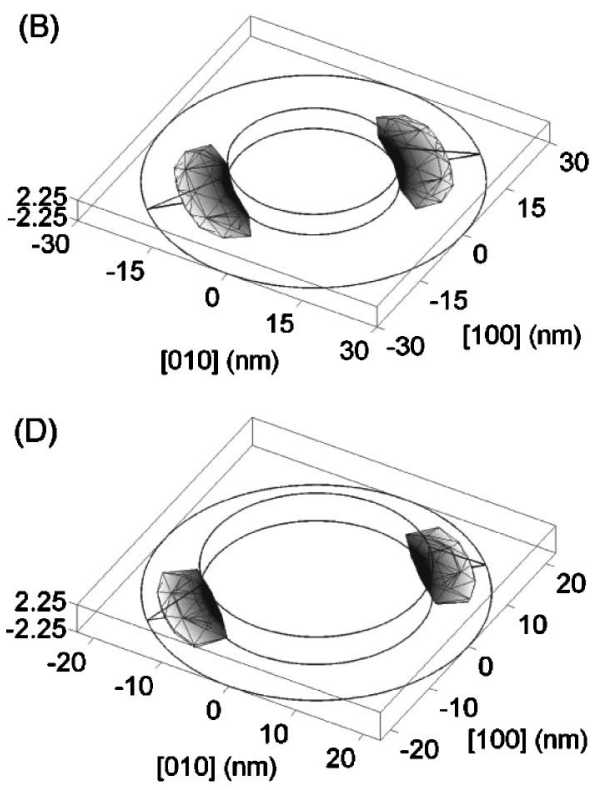

FIG. 6. Surface plots of charge-carrier probability densities $|\psi(\mathbf{r})|^{2}$ plotting the contour at $50 \%$ of the peak probability density. (a) The ground-state electron wave function for an InAs/GaAs quantum ring with outer diameter $60 \mathrm{~nm}$, inner diameter $30 \mathrm{~nm}$, and maximum height $4.5 \mathrm{~nm}$. (b) The ground-state heavy-hole wave function for the ring considered in (a). (c) The ground-state electron wave function for a ring with a similar geometry to (a) but with a $45 \mathrm{~nm}$ outer diameter. (d) The ground-state heavy-hole wave function for the ring considered in (c). radius of the ring to zero at the outer radius. Figures 6(a) and 6(b) are surface plots of the ground-state electron and hole probability densities for this structure. Both the ground-state electron and hole wave functions are localized predominantly at the inside of the ring and towards the ring base, as a consequence of the asymmetry in the ring shape. The piezoelectric potential then localizes the ground-state electron wave function on the $(1 \overline{1} 0)$ plane through the ring-center and the ground-state heavy-hole wave function on the (110) plane, as seen clearly in the figures. This structure cannot represent the real structure because the electron wave function does not propagate around the ring and also because the electron-hole overlap is far too small. However, the structure does help in understanding the origin of the electron-hole dipole moment. The magnitude of the biaxial strain varies with height for the ring profile in Fig. 5, as was previously observed for constant composition pyramids. ${ }^{29}$ At the relatively wide base, the biaxial strain is large and positive, as observed in the bulk of the symmetric rings of Fig. 1, reflecting that the InAs in the dot experiences a net compression in the $x-y$ plane and an extension in the [001] (growth) direction. Near the top however the situation tends to be reversed, so that the biaxial strain can even change sign. ${ }^{29}$ Because the heavy-hole potential includes a contribution proportional to $\varepsilon_{a x}$, the heavy-hole well is deepest at the base of the ring, tending to push the hole wave function towards the base. The piezoelectric potential then adds to this effect, localizing the hole in the (110) plane, where the piezoelectric potential maximizes the depth of the heavy-hole potential well. By contrast, the electron potential does not depend on the biaxial strain, and so the electrons do not experience an equivalent strain-induced attraction towards the ring base. However the overall ring shape causes the electron center of gravity to lie in the lower part of the ring, and the piezoelectric potential can then partly localize the electron wave function of Fig. $6(\mathrm{a})$ in the $(1 \overline{1} 0)$ plane. The overall effect of ring shape, biaxial strain, and piezoelectric potential give a calculated electron-hole dipole of the same sign as the experimental one. The calculated value $-0.3 \mathrm{~nm}$ is smaller in magnitude than the experimental result $\sim-1 \mathrm{~nm}$.

To come up with a more realistic representation of the self-assembled quantum rings, we note that for the structures in Figs. 6(a) and 6(b), the mean lateral radii calculated for the electron and hole wave functions are $20.4 \mathrm{~nm}$ and 21.0 $\mathrm{nm}$, respectively. The electron radius is considerably larger than that deduced in the capacitance spectroscopy where the field for the $s$ to $p$ transition implies an electron radius of 14 $\mathrm{nm}$. Given that the structural radius is known from the atomic force micrograph, this result on the electron radius suggests that either we are overestimating the lateral extent of the ring, or that the $\operatorname{In}_{x} \mathrm{Ga}_{1-x}$ As ring has a lateral composition gradient such that the ring is indium rich at the inner radius and more dilute at the outer radius. In the first case, a smaller outer radius tends to push the electron wave function towards the ring center. In the second case, the high indium concentration rim attracts both electrons and holes, again pulling the carrier wave functions towards the center. The possibility of an indium-rich rim has been suggested from a discussion of ring formation. ${ }^{9,12}$ We consider the possibility of a reduced outer radius in detail here as it involves fewer assumptions about the ring shape.

Figures 6(c) and 6(d) are surface plots of the ground-state electron and hole probability densities for an InAs/GaAs quantum ring with a $45 \mathrm{~nm}$ outer diameter, reduced from the $60 \mathrm{~nm}$ outer diameter in Figs. 6(a) and 6(b). The ground-state hole wave function remains pinned on the (110) plane. As before, the piezoelectric potential is strong enough to prevent the hole wave function from propagating around the ring. However, the ground-state electron wave function is more delocalized around the ring than for the larger ring. The reason for this is that the reduced ring volume weakens the piezoelectric potential, so that the localizing effects of the piezoelectric field are smaller. This structure has therefore both a large electron-hole overlap (0.7) and an extended electron wave function. Furthermore, the separation between the mean positions of the electron and hole wave functions in- 
creases to $0.5 \mathrm{~nm}$, with the same sign as in the experiments. The increase in electron-hole dipole moment is caused by the greater delocalization of the electron wave function, decreasing the overlap of the electron wave function with the piezoelectric potential maxima, and hence decreasing the extent to which the electron wave function is pulled downwards by the piezoelectric potential. Finally, the lateral radii of the electron and hole wave functions decrease to 17.4 and $17.5 \mathrm{~nm}$, respectively, so that the electron radius is reasonably consistent with the result of the capacitance spectroscopy. We find very similar results for a ring with outer diameter $60 \mathrm{~nm}$ but a radial indium concentration profile. In the terms of this discussion, this is a natural result, as also in this case, the effective ring volume is decreased.

In our calculations we inevitably retain a certain degree of symmetry whereas in the real self-assembled quantum rings, there is no reason to expect any exact rotational symmetry. The atomic force micrograph of Fig. 4 shows a clear lateral asymmetry in the ring shape. The rings are ellipsoidal, with the major axis along the [1 $\overline{1} 0]$ direction [i.e., in the (110) plane]. We saw in Figs. 3(e) and 3(f) how this tends to localize the electrons and the holes in the (110) plane, thus further increasing the magnitude of the dipole moment in a ring with sloping walls. The electrons are pushed towards the base by the piezoelectric potential in the $(1 \overline{1} 0)$ plane, as noted in Fig. 6(a). The ring asymmetry will increase the magnitude of the electron amplitude in the (110) plane, where the piezoelectric potential then acts to push the electron towards the top of the ring, and the hole towards the base. Hence, the large observed magnitude of the electron-hole in-built dipole is consistent with the AFM data on uncapped rings, which show a large asymmetry both in the vertical direction, where the ring has sloping outer walls, and also in the growth plane, where the rings are elongated in the (110) plane.

\section{CONCLUSIONS}

We have used one-band electron and hole Hamiltonians to investigate the wave functions of the ground-state electron and hole levels for nanometer-sized self-assembled quantum rings. We have considered both generic effects and also a geometry appropriate to real self-assembled rings. We find that even for a perfect self-assembled ring, the piezoelectric potential breaks the rotational symmetry, resulting in maxima in the electron confining potential with associated minima in the hole confining potential, every $180^{\circ}$ as a carrier propagates at constant radius around the ring. The consequences of this depend on the strength of the piezoelectric field. For a ring with a very strong piezoelectric potential, the electron and hole wave functions are both localized in their respective potential minima, with a small overlap between each other. For a weaker piezoelectric potential, the hole wave function is localized but, owing to the small electron mass, the electron wave function is delocalized around the ring. Finally, for very small volume rings where the piezoelectric field is small, both the electron and the hole wave functions are extended around the ring.

We have attempted to reproduce the results of spectroscopy experiments on real self-assembled quantum rings by varying the shape and composition profile of the quantum ring maintaining a consistency with AFM measurements. We find that both an asymmetric vertical profile and a radial indium concentration gradient are required to account for the experimental results. A vertical asymmetry is needed to account for the large excitonic dipole moments and a radial asymmetry is required to account for the radius of the electron wave function deduced from capacitance-voltage measurements in a magnetic field. We find that for a conical cross section and plausible radii and indium concentrations, the hole wave function is localized in the (110) plane. The hole is localized in this plane over quite a wide range of parameter space. Furthermore, the hole localization is enhanced in practice by the slight elongation of the rings (Fig. 4). However, the nature of the electron wave function depends critically on the parameters, being localized in the (1) 10$)$ plane for large volume, high indium concentration rings, extended around the ring for small volume rings, and localized in the (110) plane with the hole for rings which are significantly elongated in the (110) plane. We suggest a structure which is compatible with the spectroscopy experiments, namely a conical cross section with maximum height $\sim 5 \mathrm{~nm}$, inner radius $\sim 15 \mathrm{~nm}$, outer radius $\sim 30 \mathrm{~nm}$ with a radial indium concentration gradient, going from highindium concentration at the inner radius to small indium concentration at the outer radius.

The results do not rule out the observation of excitonic Aharanov-Bohm effects in self-assembled rings because Aharanov-Bohm effects are possibly preserved by the electron wave function which can be delocalized around the ring. However, the results demonstrate that hole localization in the (110) plane is very important. This feature is absent from all existing theories of the excitonic properties of self-assembled rings in a magnetic field. It is therefore an important task to include both piezoelectric/strain effects and excitonic effects to understand the properties of self-assembled quantum rings in a magnetic field.

\section{ACKNOWLEDGMENTS}

We would like to thank John Davies, Jorge M. Garcia, Daniel Granados, Khaled Karrai, and Axel Lorke for helpful discussions. This work was financially supported by EPSRC, UK.

\footnotetext{
${ }^{1}$ A.D. Yoffe, Adv. Phys. 50, 1 (2001).

${ }^{2}$ See, for example, D. Bimberg, M. Grundmann, and N. N. Ledentsov, Quantum Dot Heterostructures (Wiley, New York, 1998).
}

\footnotetext{
${ }^{3}$ R.J. Warburton, Contemp. Phys. 43, 351 (2002).

${ }^{4}$ S. Ruvimov, P. Werner, K. Scheerschmidt, U. Gösele, J. Heydenreich, U. Richter, N.N. Ledentsov, M. Grundmann, D. Bimberg, V.M. Ustinov, A.Yu. Egorov, P.S. Kop'ev, and Zh.I. Alferov,
} 
Phys. Rev. B 51, 14766 (1995).

${ }^{5}$ D. Leonard, K. Pond, and P.M. Petroff, Phys. Rev. B 50, 11687 (1994).

${ }^{6}$ I. Kegel, T.H. Metzger, A. Lorke, J. Peisl, J. Stangl, G. Bauer, J.M. Garcia, and P.M. Petroff, Phys. Rev. Lett. 85, 1694 (2000).

${ }^{7}$ P.W. Fry, I.E. Itskevich, D.J. Mowbray, M.S. Skolnick, J.J. Finley, J.A. Barker, E.P. O'Reilly, L.R. Wilson, I.A. Larkin, P.A. Maksym, M. Hopkinson, M. Al-Khafaji, J.P.R. David, A.G. Cullis, G. Hill, and J.C. Clark, Phys. Rev. Lett. 84, 733 (2000).

${ }^{8}$ J.M. Garcia, G. Medeiros-Ribeiro, K. Schmidt, T. Ngo, J.L. Feng, A. Lorke, J. Kotthaus, and P.M. Petroff, Appl. Phys. Lett. 71, 2014 (1997).

${ }^{9}$ R. Blossey and A. Lorke, Phys. Rev. E 65, 021603 (2002).

${ }^{10}$ D. Granados and J.M. Garcia, Appl. Phys. Lett. 82, 2401 (2003).

${ }^{11}$ A. Lorke, R.J. Lukyen, A.O. Govorov, J.P. Kotthaus, J.M. Garcia, and P.M. Petroff, Phys. Rev. Lett. 84, 2223 (2000).

${ }^{12}$ A. Lorke, R. Blossey, J.M. Garcia, M. Bichler, and G. Abstreiter, Mater. Sci. Eng., B 88, 225 (2002).

${ }^{13}$ H. Pettersson, R.J. Warburton, A. Lorke, K. Karrai, J.P. Kotthaus, J.M. Garcia, and P.M. Petroff, Physica E (Amsterdam) 6, 510 (2000).

${ }^{14}$ R.J. Warburton, C. Schäflein, D. Haft, F. Bickel, A. Lorke, K. Karrai, J.M. Garcia, W. Schoenfeld, and P.M. Petroff, Nature (London) 405, 926 (2000).

${ }^{15}$ R.J. Warburton, C. Schulhauser, D. Haft, C. Schäflein, K. Karrai, J.M. Garcia, W. Shoenfeld, and P.M. Petroff, Phys. Rev. B 65, 113303 (2002).

${ }^{16}$ D. Haft, C. Schulhasuer, A.O. Govorov, R.J. Warburton, K. Karrai, J.M. Garcia, W. Schoenfeld, and P.M. Petroff, Physica E (Amsterdam) 13, 165 (2002).

${ }^{17}$ D. Wohlleben, P. Freche, M. Esser, E. Zipper, and M. Szopa, Mod. Phys. Lett. B 6, 1481 (1992).
${ }^{18}$ W.-C. Tan and J.C. Inkson, Semicond. Sci. Technol. 11, 1635 (1996).

${ }^{19}$ A. Chaplik, Pis'ma Zh. Éksp. Teor. Fiz. 62, 885 (1995) [JETP Lett. 62, 900 (1995)].

${ }^{20}$ R.A. Römer and M.E. Raikh, Phys. Rev. B 62, 7045 (2000).

${ }^{21}$ J. Song and S.E. Ulloa, Phys. Rev. B 63, 125302 (2001).

${ }^{22}$ H. Hu, J.-L. Zhu, D.-J. Li, and J.-J. Xiong, Phys. Rev. B 63, 195307 (2001).

${ }^{23}$ I. Galbraith, F.J. Braid, and R.J. Warburton, Phys. Status Solidi A 190, 781 (2002).

${ }^{24}$ M. Bayer, M. Korkusinski, P. Hawrylak, T. Gutbrod, M. Michel, and A. Forchel, Phys. Rev. Lett. 90, 186801 (2003).

${ }^{25}$ L.A. Lavenère-Wanderley, A. Bruno-Alfonso, and A. Latgé, J. Phys.: Condens. Matter 14, 259 (2002).

${ }^{26}$ S.-S. Li and J.-B. Xia, J. Appl. Phys. 89, 3434 (2001).

${ }^{27}$ S.-S. Li and J.-B. Xia, J. Appl. Phys. 91, 3227 (2002).

${ }^{28}$ A.V. Maslov and D.S. Citrin, Phys. Rev. B 67, 121304 (2003).

${ }^{29}$ J.A. Barker and E.P. O'Reilly, Phys. Rev. B 61, 13840 (2000).

${ }^{30}$ A.D. Andreev, J.R. Downes, D.A. Faux, and E.P. O'Reilly, J. Appl. Phys. 86, 297 (1999).

${ }^{31}$ J.H. Davies, J. Appl. Phys. 84, 1358 (1998).

${ }^{32}$ M. Grundmann, O. Stier, and D. Bimberg, Phys. Rev. B 52, 11969 (1995).

${ }^{33}$ P. Offermans, P. M. Koenraad, D. Granados, and J. M. Garcia (private communication).

${ }^{34}$ J. M. Smith, P. A. Dalgarno, G. S. Buller, B. Urbaszek, G. J. Nott, R. J. Warburton, J. M. Garcia, W. Schoenfeld, and P. M. Petroff, in Proceedings of the 26th International Conference on the Physics of Semiconductors, Edinburgh, edited by A. R. Long and J. H. Davies, IOP Conf. Proc. No. 171 (Institute of Physics, London, 2002), p. 224. 